\title{
ON THE EXCHANGE POTENTIAL
}

\author{
W. J. CASPERS \\ Institute for Crystal Physics, Groningen, Netherlands
}

(Received 20 April 1964)

\begin{abstract}
A general form is derived for the socalled exchange potential. This potential gives a description of the influence of the exclusion principle on the Coulomb scattering. The diagonal elements of the potential give the well-known exchange splitting for stationary states.
\end{abstract}

WE consider the exchange interaction betwen a conduction electron and a bound electron of a metal ion. It will be shown that this interaction can be ${ }^{\prime} \cdot s$ scribed in terms of an exchange potential. The form we derive for this potential differs from that given by Kasuya [1], which is

$$
\bar{V}_{\text {ex }}\left(\vec{r}_{1}, \vec{s}_{1}, \vec{s}_{2}\right)=-J\left(\vec{r}_{1}\right) \vec{S}_{1} \cdot \vec{s}_{2} \text {. }
$$

In (1) $\vec{r}_{1}$ is the position vector of the conduction electron with respect to the ion. $\vec{S}_{1}$ and $\vec{S}_{2}$ are, respectively, the spin of the conduction electron and that of the electron in the bound state.

The theoretical expression for the exchange splitting in a stationary state suggests the following form of the potential

$$
V_{\mathrm{ex}}\left(\vec{r}_{1}, \vec{r}_{2}, \vec{S}_{1}, \vec{S}_{2}\right)=-\frac{\mathrm{e}^{2}}{\left|\vec{r}_{1}-\vec{r}_{2}\right|} P_{12}{ }^{\mathrm{r}} P_{12}{ }^{\mathrm{s}}
$$

which will be derived in the following lines. In (2) $P_{12}{ }^{r}$ and $P_{12}{ }^{s}$ respectively denote the space-exchange and the spin-exchange operators for the electron pair $(1,2)$. We like to stress that the co-ordinate $\vec{r}_{2}$ of the bound electron appears in $V_{\mathbf{e x}}$. It cannot be replaced by an expectation value because of the special form of the potential, which includes a space-exchange operator. The potential $V_{\text {ex }}$ may be useful especially for the analysis of the $s-f$ scattering in rare-earth metals $[2-8]$.

The unperturbed motion of the $s$ and $f$ electron (in general: conduction electron and bound electron) is respectively given by

$$
\begin{array}{r}
-\frac{\hbar^{2}}{2 m} \vec{\nabla}_{1}^{2} \vec{\psi}_{i}\left(\vec{r}_{1}\right)=E_{i} \bar{\psi}_{i}\left(\vec{r}_{1}\right), \\
{\left[-\frac{\hbar^{2}}{2 m} \vec{\nabla}_{2}^{2}+V_{\phi}\left(\vec{r}_{2}\right)\right] \phi_{m}\left(\vec{r}_{2}\right)=E_{m}^{\prime} \phi_{m}\left(\vec{r}_{2}\right),}
\end{array}
$$

in which $V_{\phi}$ is the (effective) potential for the $f$ electron. The effect of exchange interaction between different $f$ electrons can be separated from the scattering we are discussing. We only consider bound states $\phi_{m}$. The $\psi_{i}$ are supposed to be discrete and normalized after the introduction of boundary conditions. In this way it is possible to determine a set of states $\psi_{i}$ orthogonal to the bound states $\phi_{m}$, and asymptotically equal to the corresponding $\bar{\psi}_{i}$, apart from a normalization factor. The $\bar{\psi}_{i}$ are plane waves characterized by a wave vector $\vec{k}$. 
The antisymmetrized wave function for the electron pair in zero order including spin is given by

$$
\Phi_{i, m, M}^{ \pm}=\frac{1}{\sqrt{2}}\left[\psi_{i}\left(\vec{r}_{1}\right) \phi_{m}\left(\vec{r}_{2}\right) \pm \phi_{m}\left(\vec{r}_{1}\right) \psi_{i}\left(\vec{r}_{2}\right)\right] \mid S( \pm), M>, S(+)=0, S(-)=1, M=-S( \pm), \ldots, S( \pm) .
$$

The + and - sign respectively correspond with singlet and triplet state. The total Hamiltonian of the electron pair is given by

$$
\mathscr{H}=-\frac{\hbar^{2}}{2 m} \vec{\nabla}_{1}^{2}+V_{\phi}\left(\vec{r}_{1}\right)-\frac{\hbar^{2}}{2 m} \vec{\nabla}_{2}^{2}+V_{\phi}\left(\vec{r}_{2}\right)+\mathscr{H}^{\prime}\left(\vec{r}_{1}, \vec{r}_{2}\right), \quad \mathscr{H}^{\prime}\left(\vec{r}_{1}, \vec{r}_{2}\right)=\frac{e^{2}}{\left|\vec{r}_{1}-\vec{r}_{2}\right|},
$$

which includes the interaction between the electrons $\mathscr{P}^{\prime}$ and the perturbation of the conduction electron by the atomic potential. The matrix elements of $\mathscr{H}$ for the wave functions $\Phi$ are

$$
\int d \tau\left(\Phi_{j, n, N}^{ \pm}\right)^{+} \mathscr{H} \Phi_{i, m, M}^{ \pm}=\delta_{N M}\left[\left(E_{i}+E_{m}{ }^{\prime}\right) \delta_{j i} \delta_{n m}+\left\langle j\left|V_{\phi}\right| i>\delta_{n m}+\left\langle j, n\left|\mathscr{H}^{\prime}\right| i, m> \pm\left\langle j, n\left|\mathscr{H}^{\prime}\right| m, i>\right] .\right.\right.\right.
$$

The indices $j, n$ etc. in the right-hand side of (3) refer to the single-electron states. We have for instance

$$
\left\langle j, n\left|\mathscr{H}^{\prime}\right| i, m\right\rangle=\int d \tau_{1} d r_{2} \psi_{j}^{*}\left(\vec{r}_{1}\right) \phi_{n}^{*}\left(\vec{r}_{2}\right) \frac{e^{2}}{\left|\vec{r}_{1}-\vec{r}_{2}\right|} \psi_{i}\left(\vec{r}_{1}\right) \phi_{m}\left(\vec{r}_{2}\right)
$$

Now we want to introduce an effective Hamiltonian $\hat{\mathscr{H}}$, which gives the same matrix elements as those given in (3), for the unsymmetrized states

$$
\psi_{i}\left(\vec{r}_{1}\right) \phi_{m}\left(\vec{r}_{2}\right) \mid S_{ \pm}, M>
$$

of a pair of distinguishable particles. The \pm sign in the right-hand side of (3) may be represented by minus 1 times the spin-exchange operator $P_{12}$ s

$$
P_{12}^{s}=\frac{1}{2}\left(1+4 \vec{S}_{1} \cdot \vec{S}_{2}\right) \text {. }
$$

The space exchange operator comes in because of the reversed order of the indices $(i, m)$ in the last matrix element. Summarizing, one arrives at the following form of the effective Hamiltonian

$$
\begin{aligned}
& \hat{\mathscr{H}}=\mathscr{H}_{0}\left(\vec{r}_{1}, \vec{r}_{2}\right)+\mathscr{H}^{\prime}\left(\vec{r}_{1}, \vec{r}_{2}, \vec{S}_{1}, \vec{S}_{2}\right), \\
& \mathscr{H}_{0}\left(\vec{r}_{1}, \vec{r}_{2}\right)=-\frac{\hbar^{2}}{2 m} \vec{\nabla}_{1}^{2}-\frac{\hbar^{2}}{2 m} \vec{\nabla}_{2}^{2}+V_{\phi}\left(\vec{r}_{2}\right), \\
& \mathscr{H}^{\prime}\left(\vec{r}_{1}, \vec{r}_{2}, \vec{S}_{1}, \vec{S}_{2}\right)=V_{\phi}\left(\vec{r}_{1}\right)+\frac{e^{2}}{r_{12}}-\frac{e^{2}}{r_{12}} P_{12}{ }^{r} P_{12}{ }^{s}, r_{12}=\left|\vec{r}_{1}-\vec{r}_{2}\right| .
\end{aligned}
$$

The effect of the operator $P_{12}{ }^{r}$ is given by

$$
P_{12}{ }^{r} \psi_{i}\left(\vec{r}_{1}\right) \phi_{m}\left(\vec{r}_{2}\right)=\phi_{m}\left(\vec{r}_{1}\right) \psi_{i}\left(\vec{r}_{2}\right) .
$$

The first term in $\mathscr{H}^{\prime}$ corres ponds to scattering of the conduction electron by the atomic potential. (We neglect electron capture in this paper.) The second term represents the ordinary Coulomb interaction between both electrons, and the third term gives us the exchange potential $V_{\text {ex }}$. Normally only the diagonal
elements of $V_{\text {ex }}$ are taken into consideration it gives the same shift for triplet $\left(S(-)\right.$, and then we may drop the first term, that is $1 / 2$, in $P_{12}{ }^{s}$ because exchange splitting [9]. For the scattering 
to a space-exchange potential when multiplied by $P_{12}{ }^{r}$. The total exchange potential as given in (2) results in an interaction that is the parallel of the Heisenberg interaction in nuclear physics.

As stated before our result differs from that given by Kasuya [1] which was cited by different authors $[2,3,5-8]$. We have the opinion that Kasuya's formula (6) 1.c. should be replaced by

$$
\begin{gathered}
\Sigma_{T_{1}, T_{2}, t_{1}, t_{2}, \mu, v}\left\langle T_{1}, t_{1}\left|\frac{e^{2}}{r_{12}}\right| T_{2}, t_{2}\right\rangle a_{t_{1} v}^{+} A_{T_{1} \mu}^{+} A_{T_{2} \mu} a_{t_{2} v} \\
-\Sigma_{T_{1}, T_{2}, t_{1}, t_{2}, \mu, v}\left\langle T_{1}, t_{1}\left|\frac{e^{2}}{r_{12}}\right| t_{2}, T_{2}\right\rangle a_{t_{1} v}^{+} A_{T_{1} \mu}^{+} A_{T_{2} v} a_{t_{2}} \mu,
\end{gathered}
$$

and taking into account both terms one arrives at exactly the same interaction as given in our formula (4).

One cannot expect that the exchange interaction may be represented adequately by an interaction of the form introduced by Kasuya, 1.c. formula (12), even if one drops the term $1 / 2$ in $P_{12}{ }^{s}$. We have tried to equate corresponding matrix elements in order to derive a functional form for the potential $J(r)$. The troubles we run into strongly suggest an inconsistency in Kasuya's approach. His approximation, in which $J\left(t, t^{\prime}\right)$ or $J\left(\vec{k}, \vec{k}^{\prime}\right)$ is replaced by $J\left(\left|\vec{k}-\vec{k}^{\prime}\right|\right)$ is not warranted, as follows from a detailed examination of formula (8) 1.c. Evaluating matrix elements of $\mathscr{K}^{\prime}$, given in formula (4), in order to compute scattering cross sections, one may use in first approximation plane waves for the states $\vec{k}$ and $\vec{k}^{\prime}$.

Our result is quite general and may be used also for ions with more than one $f$ electron. For these ions the coupling between the different orbital momenta and spins, together with the spin-orbit coupling, have to be taken into account explicitly for a correct description of the scattering.

For a state with zero orbital momentum and total spin $S$ one may derive another form of the potential on the basis of (2), if only elastic scattering is considered $(\Delta S=0)$

$$
V_{\mathrm{ex}}=\Sigma_{i} \frac{e^{2}}{\left|\overrightarrow{\boldsymbol{r}}_{1}-\vec{r}_{i}\right|}\left[1-\frac{1}{2} P_{1 i}^{r}\left(1+a(S) \vec{S}_{1} \cdot \vec{S}\right)\right] .
$$

In this expression $i$ numbers the different $f$ electrons and $\vec{S}$ is the total spin momentum operator. The value of the constant $a(S)$, which depends on the spin quantum number, follows from the general theory of addition of angular momenta, as discussed, for instance, by Condon and Shortley [10]. The same form of $V_{\text {ex }}$ may be used for the case of a quenched orbital momentum, which does not apply to the rare-earth metals. Our formula (5) may be compared with the potential used in refs. $[2,3,6,7,8]$.

For the other case, in which the orbital motion of the $f$ electrons has to be taken into account explicitly, we do not expect that the exchange potential, as given by a sum of terms of the form (2), 'can be simplified in an analogous way. It is most unlikely that a simple form in terms of $\vec{r}_{1}, \vec{r}_{i}, \vec{s}$ and $\vec{J}$ can be found for (5), $\vec{J}$ being the operator of the total angular momentum of the ion.

So far we have not analyzed in detail the consequences of our result (2) for the value of the cross section of the spin-dependent scattering in special cases. This will be the object of further studies, especially for the rare-earth metals.

\section{Acknowledgement}

The author acknowledges discussions with Professor A. J. Dekker, which led to some improvements of the text of this paper.

This work is part of the research program of the "Stichting voor Fundamenteel Onderzoek der Materie" (Foundation for F undamental Research on Matter - F.O.M.) and was made possible by financial support from the "Nederlandse Organisatie voor Zuiver Wetenschappelijk Onderzoek" (Netherlands Organization for the Advancement of Pure Research - Z.W.O.).

\section{References}

1. T. KASUYA, Progr. Theor. Phys. 16, 45 (1956).

2. K. YOSIDA, Phys. Rev. 107, 396 (1957). 
3. P. G. DE GENNES and J. FRIEDEL, J. Phys. Chem. Solids 4, 71 (1958).

4. G. S. ANDERSON and S. LEGVOLD, Phys. Rev. Letters 1, 322 (1958).

5. P. G. DE GENNES, C. R. Acad. Sci., Paris 247, 1836 (1958).

6. R. BROUT and H. SUHL, Phys. Rev. Letters 2, 387 (1959).

7. T. VAN PESKI - TINBERGEN and A. J. DEKKER, Physica 29, 917 (1963).

8. S. H. LIU, Phys. Rev. 132, 589 (1963).

9. J. H. VAN VLECK, The Theory of Electric and Magnetic Susceptibilities p. 316. Oxford University Press, (1932).

10. E. U. CONDON and G. H. SHORTLEY, The Theory of Atomic Spectra Chap. III. Cambridge University Press (1963).

Editor's Note: Dr. Caspers' point is well taken. It is widely accepted and understood in band theory that the Fock exchange potential is non-local (see the discussion in P. W. Anderson, Concepts in Solids, p. 53ff, and references therein), although the Slater approximation is often used in actual calculations. Correspondingly, the exchange potential in the Dirac - van Vleck vector model must be non-local, although it has hitherto always been treated as local. It will be interesting to see whether this result has measurable consequences, and also to search for other formal expressions for the effect.

P. W. A. 